\title{
Dispositivo de alineación de muestras para el difractómetro de rayos $X$ con control de posición e interfaz de manipulación
}

\author{
Device for sample alignment for $x$-ray difractometer with position control and \\ handling interface
}

\section{Dispositivo de alienação de amostras para o difractómetro de raios $\mathrm{x}$ com controle de posição e interfase de manipulação}

Daniel Santiago Jiménez-Novoa ${ }^{1}$, Johann Sebastián González-Bustamante ${ }^{2}$, William Aperador-Chaparro ${ }^{3}$

Forma de citar: D.S. Jiménez-Novoa, J.S. González-Bustamante y W. Aperador-Chaparro, "Dispositivo de alineación de muestras para el difractómetro de rayos X con control de posición e interfaz de manipulación", Respuestas, vol. 22, no. 1, pp. 84-95, 2017.

Recibido:

Agosto 16 de 2016

Aceptado:

Diciembre 1 de 2016

\section{Resumen}

En este artículo se detalla el diseño y fabricación de un prototipo mecánico que actúa como soporte de distintos tamaños de muestras para el análisis de difracción de rayos X, que controla la posición de la probeta, logrando una orientación precisa con respecto al haz de rayos X, esto beneficia al sector industrial en el desarrollo de nuevos proyectos con materiales al mejorar el desempeño de los mismos. El dispositivo requiere de la integración de distintos elementos mecánicos, electrónicos junto con conocimientos en el área programación para su correcto funcionamiento con el alto grado de precisión necesario para garantizar la posición de la muestra, a partir del diseño y la buena elección de los materiales en la fabricación. Se logró garantizar la precisión de la posición de la muestra a evaluar, por lo tanto, las partes mecánicas a utilizar en el prototipo deben ser de la mayor precisión que podamos obtener en este tipo de elementos según las medidas de la estructura.

Palabras clave: Difracción de rayos X, diseño, electrónica, precisión.

\begin{abstract}
This article describes the design and manufacture of a mechanical prototype which acts to support different sizes of samples for the analysis of X-ray diffraction, which controls the position of the cylinder, achieving an accurate orientation with respect to X-ray beam, which benefits the industry in the development of new projects with materials that improve their performance. The device requires the integration of various mechanical, electronic elements along with expertise in the field of programming for proper operation with high degree of accuracy that guaranties the position of the sample, from design and good choice of fabrication materials. We managed to ensure the accuracy of the sample position; therefore the mechanical parts used in the prototype should be as accurately as we can get in this kind of items as measured by the structure.
\end{abstract}

Keywords: X-Ray Diffraction, design, electronic, accuracy. 


\section{Resumo}

Neste artigo detalha-se o design e fabricação de um protótipo mecânico que atua como suporte de diferentes tamanhos de amostras para a análise de difração de raios X, que controla a posição da proveta, logrando uma orientação precisa com respeito ao feixe de raios $\mathrm{X}$, isto beneficia ao sector industrial no desenvolvimento de novos projetos com materiais ao melhorar o desempenho dos mesmos. O dispositivo requer da integração de diferentes elementos mecânicos, electrónicos junto com conhecimentos na área de programação para seu correto funcionamento com o alto grau de precisão necessário para garantir a posição da amostra, a partir do design e a boa eleição dos materiais na fabricação. Logrou-se garantir a precisão da posição da amostra a avaliar, portanto, as partes mecânicas a utilizar no protótipo devem ser da maior precisão que podamos obter neste tipo de elementos segundo as medidas da estrutura.

Palavras-chave: Difração de raios X, design, electrónica, precisão.

\section{Introducción}

El estudio y análisis de materiales por difracción de rayos X proporciona información detallada de la estructura cristalográfica en estado sólido de las muestras de compuestos orgánicos e inorgánicos [1]-[5]. Las diferentes aplicaciones que ofrece el difractómetro de rayos, gracias a los análisis que se realizan a los materiales, son de gran importancia para varios sectores de la industria que operan con materiales, de la misma forma, para su investigación [6]-[9].

Debido a los diferentes tamaños de las muestras se requiere un porta muestras que soporte y pueda corregir su posición tanto vertical como horizontalmente, siendo ésta ubicada en el punto central donde va a entrar en contacto con el haz de rayos X, al llegar a este punto la muestra debe estar totalmente equilibrada para que exista una orientación precisa y se difracte el haz de rayos $\mathrm{X}$ correctamente a la ubicación que brinda los resultados esperados, los cuales se deben observar desde el computador [10]-[12].

El dispositivo requiere de la integración de distintos elementos mecánicos y electrónicos junto con conocimientos en el área de control y programación para su correcto funcionamiento con el alto grado de precisión necesario para garantizar la posición de la muestra, a partir del diseño y la buena elección de los materiales en la fabricación [13]-[18].

Los dispositivos que han sido diseñado de acuerdo con las medidas del espacio libre que tenga cada uno de los difractómetro de rayos $\mathrm{X}$ bajo el punto central de funcionamiento, se procede a diseñar el sistema de movimiento en los ejes X y Z, donde se realizan distintos tipos de montajes mediante CAD [19]. Se determinan mediante cálculos las fuerzas a realizar sobre la estructura sabiendo la carga que van a manejar los compontes, de esta forma el torque necesario para encontrar los motores adecuados para mover el sistema diseñado.

Los dispositivos deben ser diseñados mediante programas especializados en simulación, donde se le asignan las fuerzas, los apoyos y momentos, el cual indican resultados en estudios de flexión y deformación, de esta forma poder elegir el mejor, más preciso y optimo modelo de funcionamiento según requerimientos. Se debe buscar a partir de sus propiedades el material que mejor se acople a las condiciones necesarias de diseño respecto 
Dispositivo de alineación de muestras para el difractómetro de rayos X con control de posición e interfaz de manipulación

a la densidad, dureza y resistencia según las condiciones del ambiente donde se va a operar con el dispositivo. Las posiciones de forma correcta se garantizan mediante los motores paso a paso que están controlados mediante drivers. La activación y manipulación de estos motores se va a dar mediante una interfaz manejada a partir de un dispositivo móvil en forma de aplicación, de esta forma es fácil de adquirir y utilizar por la persona que manipule el dispositivo [20]-[21].

El presenta trabajo se diseña y construye un dispositivo y se necesita acondicionar uno que cumpla con los requerimientos para el correcto funcionamiento del difractómetro de rayos $\mathrm{X}$, de esta forma se garantiza una mejor y más confiable información acerca de las muestras de materiales para su estudio y análisis.

\section{Materiales y métodos}

Primero se evaluaron los puntos principales necesarios para el diseño del dispositivo, de tal forma que cumpliera con todos los requerimientos.

Ergonomía. El diseño del dispositivo se hace teniendo en cuenta el espacio de trabajo en el que se desplaza el porta muestras, igualmente teniendo cuenta las necesidades del operario se facilita la forma de manipulación del dispositivo mediante una interfaz de dispositivo móvil haciendo así más amigable la interacción usuario - máquina en el ambiente adecuado de trabajo donde se encuentra el difractómetro de rayos X. De esta forma se brinda un ambiente de trabajo más cómodo y confortable para el operario sin complicaciones de salud, generándole un mejor desempeño en el trabajo que realice con el dispositivo.

Estética. La estética del prototipo se diseñó para que se adaptara al entorno en el cual trabaja, siendo el interior del difractómetro de rayos $\mathrm{X}$, donde la mayoría de tonalidades son claras, por lo que el color predominante en el dispositivo es el color blanco para continuar con este patrón. La textura final del dispositivo se diseñó de tal forma que fuese agradable para el operario, debido a que su acoplamiento y extracción pueda realizarse de manera sencilla y eficaz para intercambiar entre los diferentes dispositivos disponibles en el laboratorio.

Funcionamiento. El dispositivo debe cumplir con las especificaciones requeridas para un estudio más eficaz de las muestras a evaluar en el difractómetro de rayos $\mathrm{X}$ garantizando su funcionamiento con un adecuado diseño, con componentes mecánicos y electrónicos de calidad y cálculos previos con estudios de simulación que demuestre su correcta selección.

Peso. El peso final del dispositivo es liviano para evitar una carga alta directamente en el eje donde va acoplado y así pueda permanecer tiempo completo en el difractómetro de rayos $X$. Otra ventaja de mantener el dispositivo con un bajo peso es la facilidad del desplazamiento y poder seleccionar motores más precisos, de bajo peso, tamaño y solicitud de energía.

Desplazamiento de los ejes. Debido a que las probetas y muestras empleadas en los análisis realizados por el grupo de investigación en el difractómetro de rayos $\mathrm{X}$, se determinó dejar un movimiento horizontal máximo de $5 \mathrm{~cm}$ y un movimiento vertical máximo de $5 \mathrm{~cm}$, el cual permite ubicar todos los tipos de muestras disponibles actualmente en el laboratorio.

\section{Selección material - disponibilidad.} Teniendo en cuenta las características que debe tener el dispositivo en cuanto a peso y firmeza, buscando un material con una densidad baja y con alta dureza, se tienen en cuenta distintos tipos de materiales como el aluminio teniéndolo en cuenta como la opción más viable en caso de materiales metálicos y 
en el caso de polímeros se opta por el Empack cuyas propiedades son las más adecuadas para este proyecto, siendo este un material más fácil de manipular en cuanto a los procesos necesarios para realizar cada una de las piezas, de igual forma es un material con alta dureza y una densidad baja, éste material es recomendado para superficies de desgaste ya que cuenta con un bajo coeficiente de fricción y es resistente al impacto.

Luego de evaluar las propiedades tanto del aluminio como del Empack se optó por el material polimérico ya que es la mejor opción en cuanto al uso que se le va a dar a éste material en el dispositivo de alineación de muestras, este material se puede adquirir fácilmente y en cantidades variadas para evitar el desperdicio de material.

En la figura 1, se procede a realizar un diseño asistido por computadora que permita realizar los dos movimientos lineales necesarios para el posicionamiento de la muestra, incluyendo las partes mecánicas a implementar y los dos motores que realizaran el movimiento de la siguiente forma:

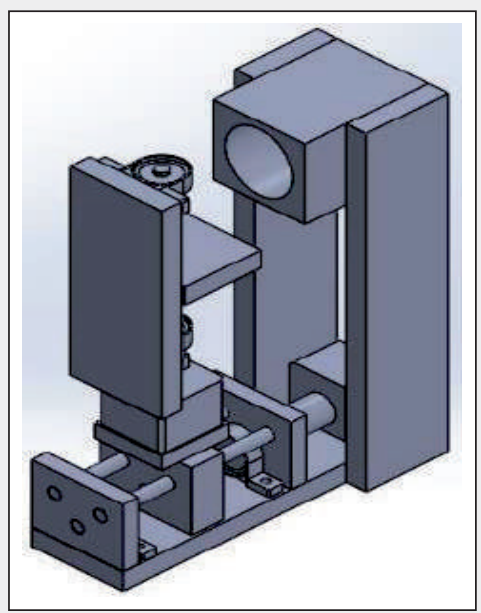

Figura 1. Diseño final del dispositivo. Fuente: Autores

\section{Resultados y análisis}

\subsection{Análisis estudio de Diseño asistido por computadora (CAD)}

En la figura 2, se observa el ensamblaje del dispositivo final se le asignan las caras de las piezas donde se indican las sujeciones en el eje del difractometro de rayos $\mathrm{X}$ en el cual es acoplado el dispositivo y las cargas en la plataforma donde se posicionaran las muestras que va a manejar el dispositivo.

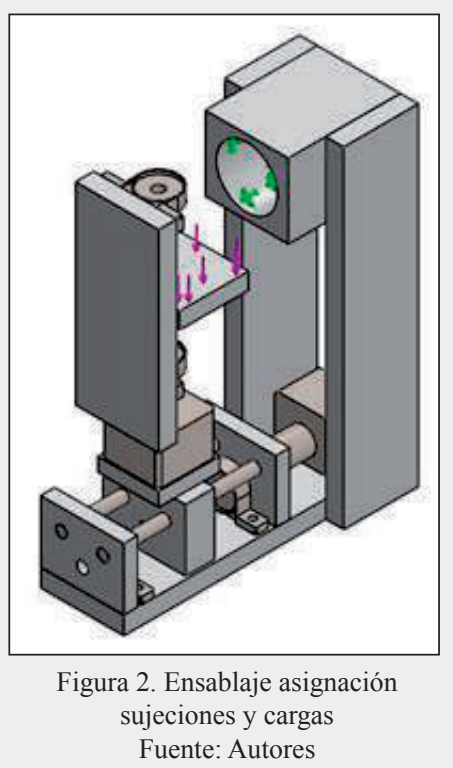

En la figura 3 se representa el análisis el cuál arroja un gran resultado en las tensiones, donde se puede apreciar que los ejes del movimiento horizontal reciben la carga del movimiento vertical, permitiendo que el tornillo que realiza el desplazamiento no sea sometido a una carga alta y asi el motor tenga la fluidez deseada a diferentes velocidades y no se pierda precision en su movimiento. Tambien nos permite determinar que el material Empack no tendra problema para realizar la estructura y tendra la estabilidad necesaria para mantener una orientacion precisa entre la muestra y el haz de rayos X. 
Dispositivo de alineación de muestras para el difractómetro de rayos X con control de posición e interfaz de manipulación

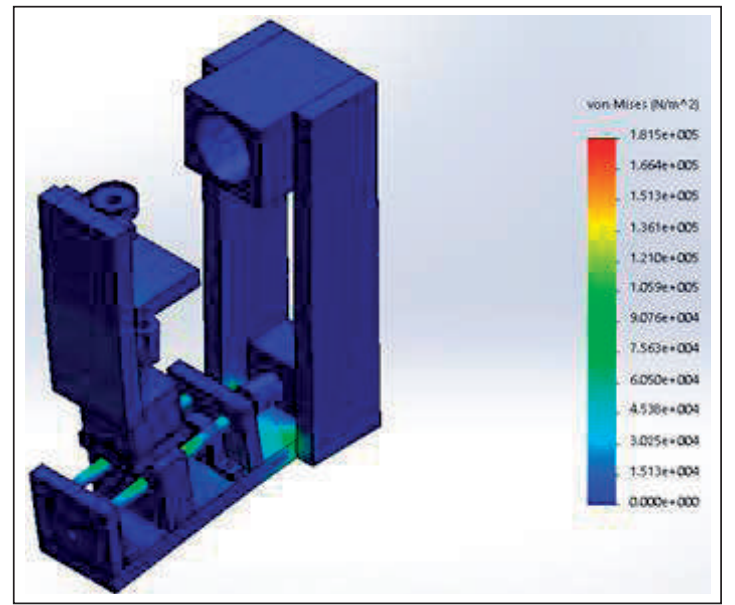

Figura 3. Análisis de tensiones en el dispositivo. Fuente: Autores

Con los estudios y resultados obtenidos del diseño $\mathrm{CAD}$ escogido se realiza la construcción de cada una de las piezas del dispositivo con las medidas adecuadas para el espacio de trabajo que tiene el difractómetro de rayos $\mathrm{X}$, este diseño consta de tres partes fundamentales para su funcionamiento mecánico junto con su respectiva parte electrónica y circuito impreso que se encarga de garantizar la correcta posición del soporte de la muestra.

En la figura 4, se observa el acople del dispositivo con el eje principal del difractómetro de rayos X; el cual va a soportar todo el peso del dispositivo con la muestra a evaluar, motores y componentes electrónicos, que, con los resultados obtenidos en estudio de deformación con el material escogido, en este caso Empack, cumple con los requerimientos para que no presente ningún tipo de falla.

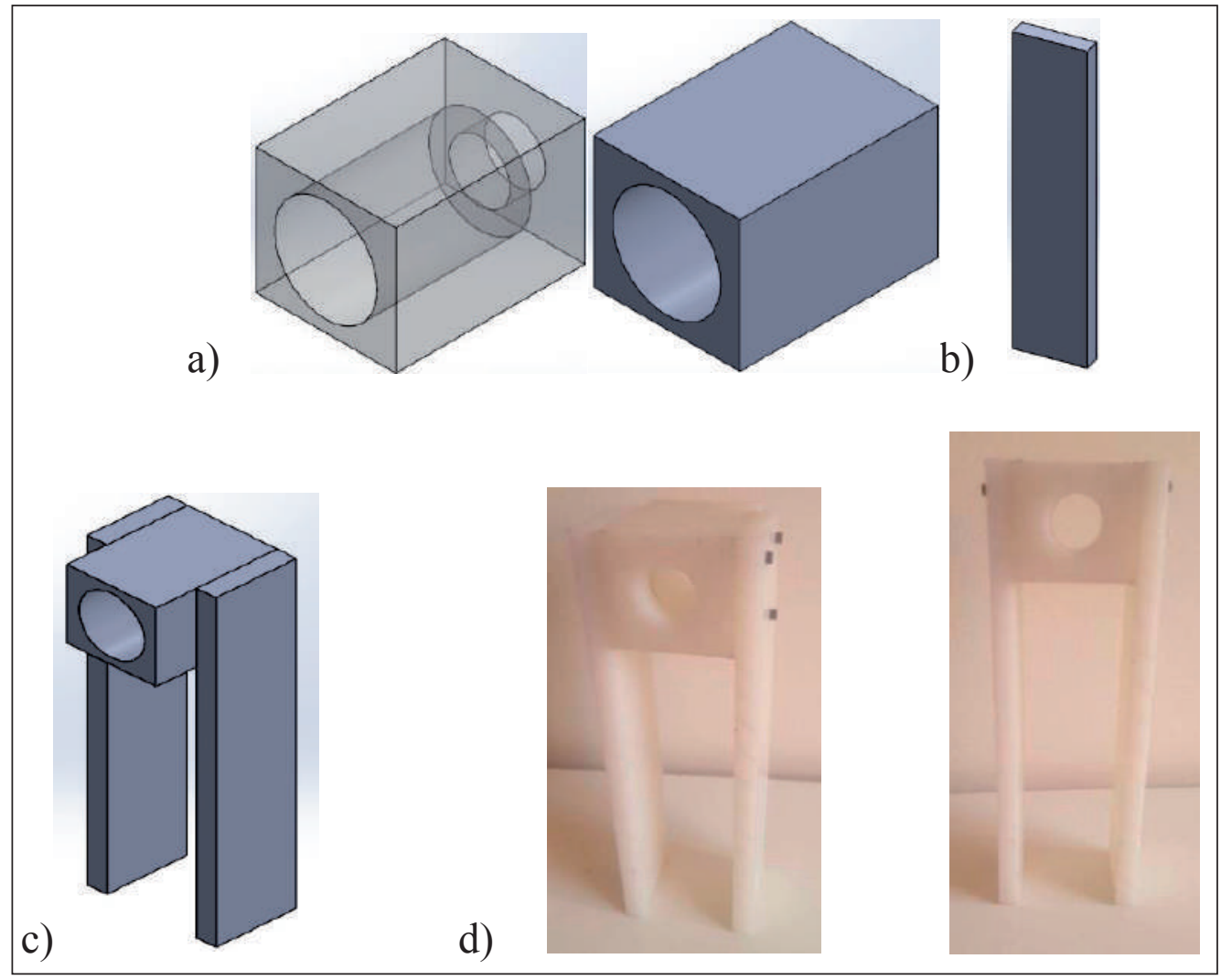

Figura 4. Acople con el eje del difractómetro (a) Acople eje, b) Laterales soporte,

c) Ensamble acople, d) Construcción acople)

Fuente: Autores 
La segunda parte fundamental del dispositivo es el eje $\mathrm{X}$ o eje horizontal, el que se encuentra ubicado fijamente en la parte inferior del acople con el eje del difractómetro de rayos $\mathrm{X}$, éste tiene un mecanismo de tuerca tornillo que da la precisión requerida, este mecanismo tiene chumaceras y piezas donde quedan de forma fija unas barras en las cuales se va a deslizar, gracias a dos rodamientos lineales, la pieza en la cual se va a montar el eje vertical con su respectivo motor (figura 5).

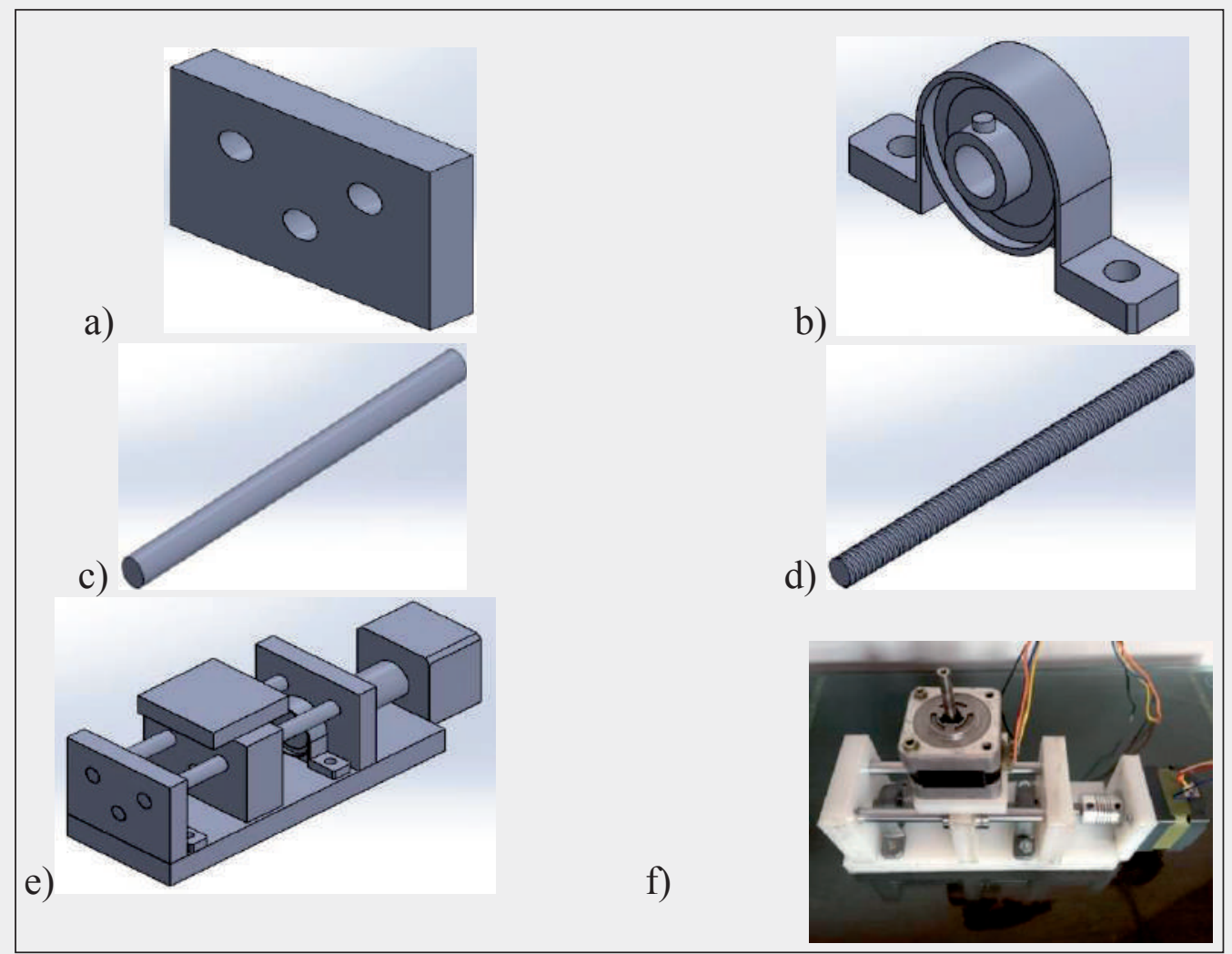

Figura 5. Eje horizontal (a) Soporte ejes de movimiento, b) Chumacera, c) eje de deslizamiento, d) tornillo, e) Ensamblaje eje horizontal CAD, f) Ensamblaje eje horizontal construcción. Fuente: Autores

En la figura 6, se observa el tercer mecanismo del dispositivo es el eje vertical o eje $\mathrm{Z}$, quien se va a encargar de ubicar la altura del porta muestras en el punto exacto donde llega es haz de rayos $\mathrm{X}$. Este mecanismo, de la misma forma que el eje horizontal, consta del sistema tuerca tornillo y las dos chumaceras, en este caso sin las barras metalizas ya que no hay 


\section{Respuestas}

Cúcuta-Colombia Vol. 22 No. 1

Enero - Junio 2017 ISSN 0122-820X E-ISSN 2422-5053 PP: 84-95

Dispositivo de alineación de muestras para el difractómetro de rayos $\mathrm{X}$ con control de posición e interfaz de manipulación

a)

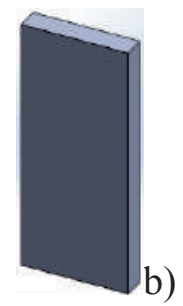

d)
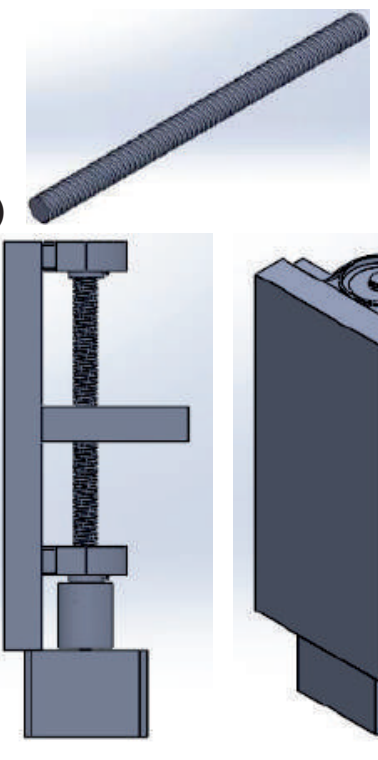

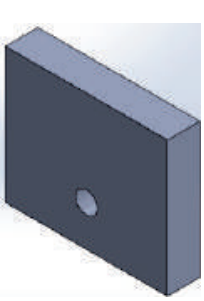

e)

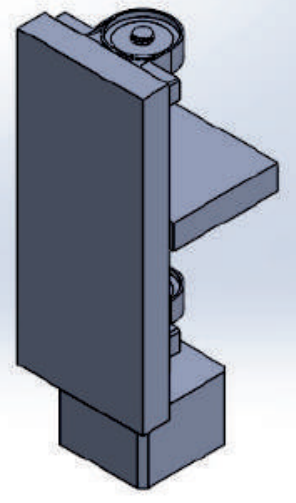

c)

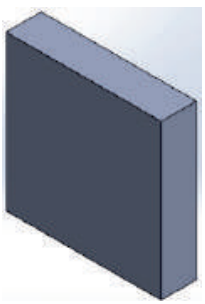

g)

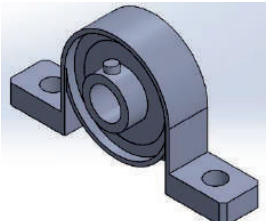

Figura 6. Eje vertical (a) Soporte mecanismo eje vertical, b) porta muestras, c) Soporte motor, d) Tornillo, e) Chumaceras, f) Ensamblaje eje vertical CAD, g) Ensamblaje eje vertical construcción: Fuente: Autores

En la figura 7 se observa cada uno de los ejes del dispositivo se va a mover con un motor paso a paso, el cual trasmite su movimiento al tornillo por medio de un acople flexible.

a)

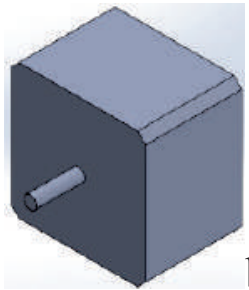

b)

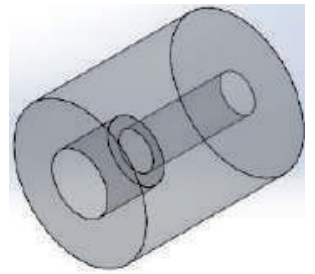

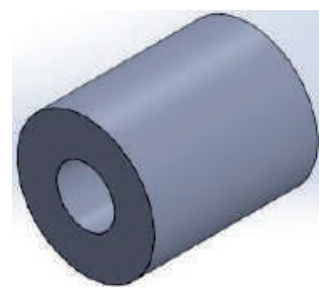

c)

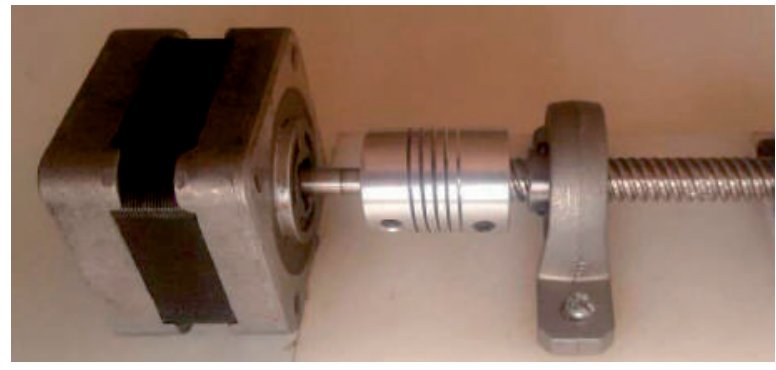

Figura 7. Motores paso a paso con acople (a) Motor paso a paso, b) Acople motor-Tornillo, c)

Construcción motor-acople.

Fuente: Autores 
En la figura 8 se observa el acople de las tres partes o componentes principales se obtiene el producto final, el dipositivo de alineación de muestras, listo para funcionar.

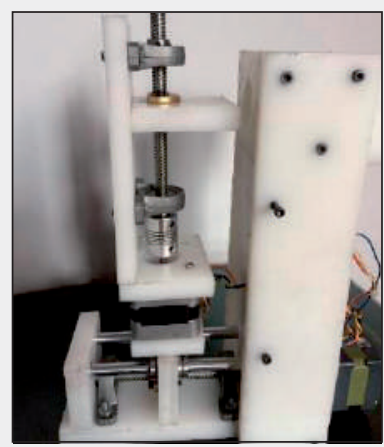

Figura 8. Ensamblaje final del dispositivo construido. Fuente: Autores

\subsection{Interfaz con dispositivos móviles}

Se realiza una interfaz para dispositivos móviles Android sencilla de utilizar y fácil de instalar, sirve para controlar la posición deseada de la muestra teniendo en cuenta los pasos de los motores, los cuales transmiten el movimiento al eje X y al eje $\mathrm{Z}$. Se realiza a través de tecnología Bluetooth (figura 9).

Esta interfaz es totalmente amigable para el operador ya que consta de sencillos botones para encender y detener el dispositivo, junto con los que indican la dirección a donde se desea mover la muestra.

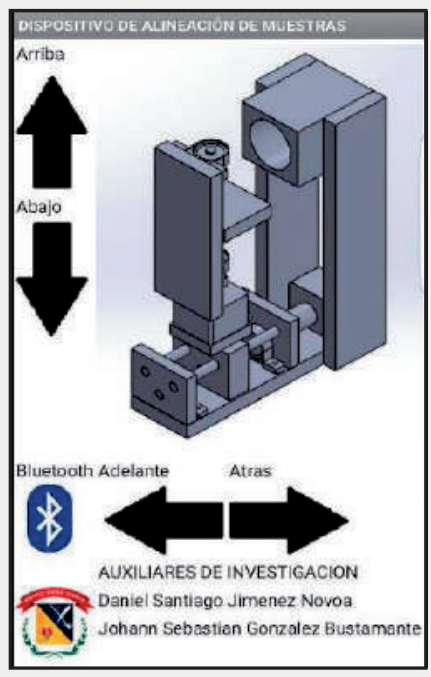

Enero - Junio 2017

Figura 9. Interfaz para dispositivos móviles.

Fuente: Autores

\subsection{Difractograma con el dispositivo y sin él.}

Para tener un adecuado nivel óptico del difractómetro, se implementa un medidor de caratula con precisión de micras, el cual es posicionado y calibrado a nivel superficial de la muestra sin el dispositivo desarrollado, esto con el fin de obtener el punto en el cual debe quedar la muestra. Posteriormente, se realiza el montaje del dispositivo para proceder a realizar la medición de la muestra de silicio. Con el fin de contrastar la medición del equipo se realiza el ensayo a la muestra de silicio con y sin el dispositivo, los difractogramas se presentan en la figura 10. 


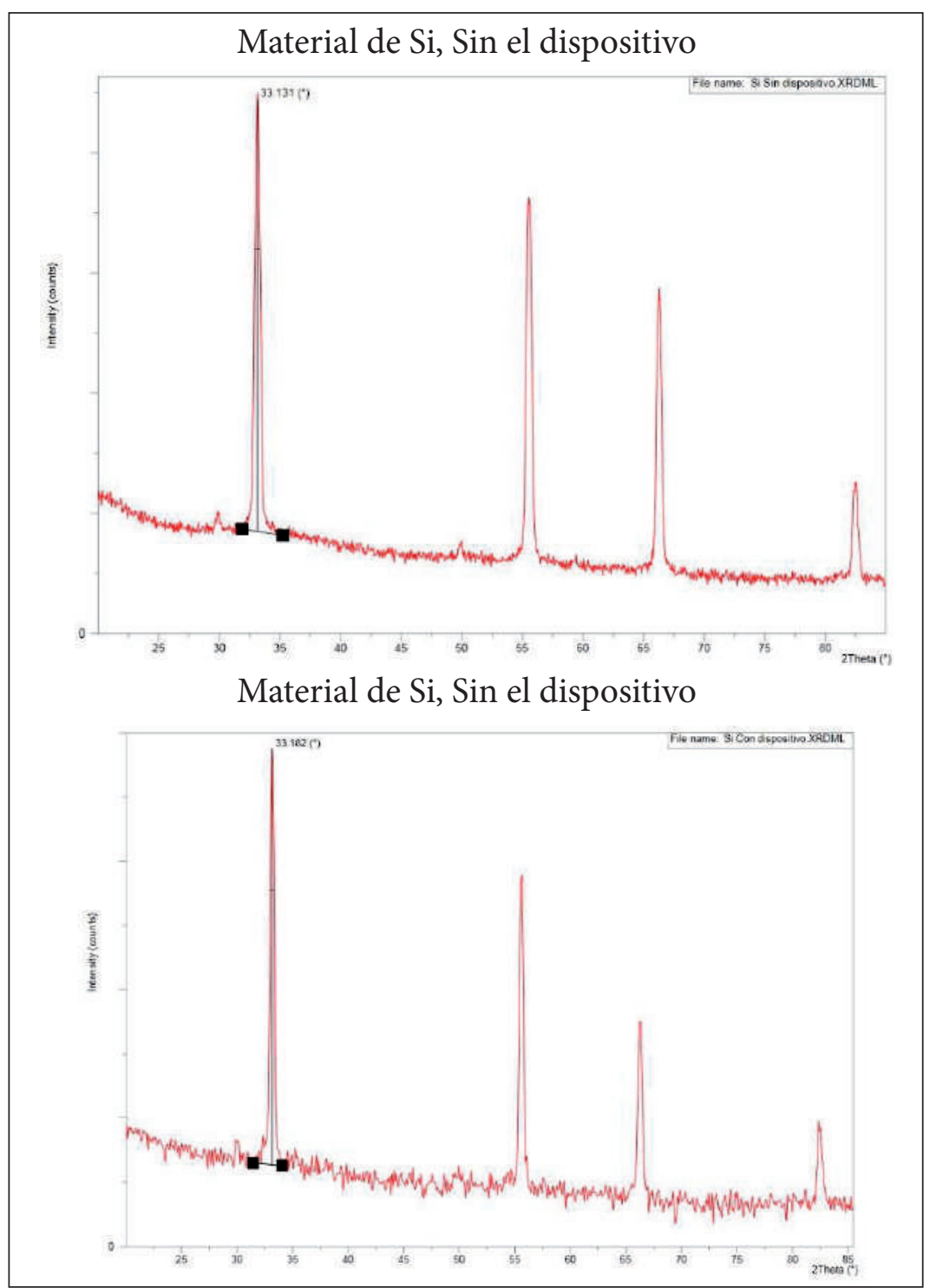

Figura 10. Difractogramas de Silicio con y sin el dispositivo. Fuente: Autores

La mayor reflexión del silicio medida con un tubo de cobalto debe presentar en 33.152, al realizar la medición de la muestra de calibración sin el dispositivo la reflexión se presentó 33.131, mientras que la medición realizada a la muestra con el dispositivo desarrollado se presentó en 33.182, lo que indica que pese a que existe un corrimiento mínimo, el dispositivo permite obtener valores muy cercanos a los esperados.

\section{Conclusiones}

El dispositivo final posiciona muestras de diferentes tamaños con una orientación precisa y se da la estabilidad necesaria de los tipos de muestras que se procede a analizar, permitiendo que el estudio por difracción de rayos $\mathrm{X}$ se logré a su totalidad con la incidencia exacta del haz de rayos $\mathrm{X}$.

El movimiento mecánico del dispositivo demuestra fluidez con los diferentes tamaños y tipos de muestra, donde no se presentan fallos en la estructura ni en la parte electrónica, la cual trabaja en muy buenas condiciones.

La aplicación para dispositivos móvil es de 
fácil instalación, y su uso permite una sencilla y eficaz interacción entre el usuario y el dispositivo, realizando los movimientos en un tiempo de respuesta mínimo y una interacción por bluetooth de fácil conexión.

El desarrollo del dispositivo es de gran ayuda para los análisis y proyectos desarrollados por el grupo de investigación, reemplazando el resorte incluido en el difractómetro de rayos $\mathrm{X}$ por un posicionamiento preciso, orientado $\mathrm{y}$ con posibilidad de examinar un rango muy amplio de nuevas muestras.

\section{Agradecimientos}

Los autores agradecen a la Vicerrectoría de Investigaciones de la Universidad Militar Nueva Granada por la financiación de este trabajo, derivado del proyecto ING2100 vigencia 2016.

\section{Referencias}

[1] L.D. Cussen and K. Lieutenant, "Computer simulation tests of optimized neutron powder diffractometer configurations", Nuclear Instruments and Methods in Physics Research Section A: Accelerators, Spectrometers, Detectors and Associated Equipment, vol. 822, pp. 97-111, 21 June 2016.

[2] L.D. Cussen, "Optimizing constant wavelength neutron powder diffractometers", Nuclear Instruments and Methods in Physics Research Section A: Accelerators, Spectrometers, Detectors and Associated Equipment, vol. 821, pp. 122-135, 11 June 2016.

[3] C. Randau, H.G. Brokmeier, W.M. Gan M. Hofmann, et. al, "Improved sample manipulation at the STRESSSPEC neutron diffractometer using an industrial 6-axis robot for texture and strain analyses", Nuclear Instruments and Methods in Physics Research Section A: Accelerators, Spectrometers, Detectors and Associated Equipment, vol. 794, pp. 67-75, 11 September 2015.

[4] J. Décobert, R. Guillamet, C. Mocuta, G. Carbone, and H. Guerault, "Structural characterization of selectively grown multilayers with new high angular resolution and sub-millimeter spotsize x-ray diffractometer", Journal of Crystal Growth, vol. 370, pp. 154-156, 1 May 2013.

[5] H. Hong and T.C. Chiang, "A six-circle diffractometer system for synchrotron $\mathrm{X}$-ray studies of surfaces and thin film growth by molecular beam epitaxy", Nuclear Instruments and Methods in Physics Research Section A: Accelerators, Spectrometers, Detectors and Associated Equipment, vol. 572, no. 2, pp. 942-947, 11 March 2007.

[6] C. Niu, X. Liu, J Meng, L. Xu, M. Yan, et al, "Three dimensional V2O5/ NaV6O15 hierarchical heterostructures: Controlled synthesis and synergistic effect investigated by in situ X-ray diffraction", Nano Energy, vol. 27, pp. 147-156, September 2016.

[7] S. Argast, and T Corey, "Using the World Wide Web for interactive control of an X-ray diffractometer", Computers \& Geosciences, vol. 24, no. 7, pp. 633640, August 1998.

[8] T. Nakano, K. Kaibara, Y. Tabata, N. Nagata, et al, "Unique alignment and texture of biological apatite crystallites in typical calcified tissues analyzed by microbeam $\mathrm{x}$-ray diffractometer system", Bone, vol. 31, no. 4, pp. 479487, October 2002.
Enero - Junio 2017 ISSN 0122-820X

E-ISSN 2422-5053 PP: 84-95 
[9] T. Kawasaki, T. Nakamura, K. Toh, T. Hosoya, et al, "Detector system of the SENJU single-crystal time-of-flight neutron diffractometer at J-PARC/ MLF", Nuclear Instruments and Methods in Physics Research Section A: Accelerators, Spectrometers, Detectors and Associated Equipment, vol. 735, pp. 444-451, 21 January 2014.

[10] J. Altenkirch, A. Steuwer, P.J. Withers, T. Buslaps, et al "Robotic sample manipulation for stress and texture determination on neutron and synchrotron X-ray diffractometers", Nuclear Instruments and Methods in Physics Research Section A: Accelerators, Spectrometers, Detectors and Associated Equipment, vol. 584, no. 2-3, pp. 428-435, 11 January 2008.

[11] Y.A. Gaponov, E.A. Dementyev, D.I. Kochubei, and B.P. Tolochko, "Portable high precision small/wide angle X-ray scattering diffractometer", Nuclear Instruments and Methods in Physics Research Section A: Accelerators, Spectrometers, Detectors and Associated Equipment, vol. 467-468, pp. 1092-1096, 21 July 2001.

[12] L.L. Fan, S. Chen, Q.H. Liu, G.M. Liao, et al, "The epitaxial growth and interfacial strain study of $\mathrm{VO} 2 / \mathrm{MgF} 2$ (001) films by synchrotron based grazing incidence X-ray diffraction", Journal of Alloys and Compounds, vol. 678, pp. 312-316, 5 September 2016.

[13] W. Kirchmeyer, O. Grassmann, N. Wyttenbach, J. Alsenz, et al, "Miniaturized X-ray powder diffraction assay (MixRay) for quantitative kinetic analysis of solvent-mediated phase transformations in pharmaceutics", Journal of Pharmaceutical and

[16] D.V. Rao, G.E. Gigante, Y.M. Kumar, R. Cesareo, et al, "Synchrotronbased crystal structure, associated morphology of snail and bivalve shells by X-ray diffraction", Radiation Physics and Chemistry, vol. 127, pp. 155-164, October 2016.

[17] Y. Hattori and M. Otsuka, "Analysis of the stabilization process of indomethacin crystals via $\pi-\pi$ and $\mathrm{CH}-\pi$ interactions measured by Raman spectroscopy and X-ray diffraction", Chemical Physics Letters, vol. 661, pp. 114-118, 16 September 2016.

[18] S.R. Kada, P.A. Lynch, J.A. Kimpton and M.R. Barnett, "In-situ X-ray diffraction studies of slip and twinning in the presence of precipitates in AZ91 alloy", Acta Materialia, vol. 119, pp. 145-156, 15 October 2016.

[19] P. Muthuraja, M. Sethuram, T. Shanmugavadivu and M. Dhandapani, "Single crystal X-ray diffraction 
and Hirshfeld surface analyses of supramolecular assemblies in certain hydrogen bonded heterocyclic organic crystals", Journal of Molecular Structure, vol. 1122, 146-156, 15 October 2016.

[20] A. Fasihizad, A. Akbari, M. Ahmadi, M. Dusek et al, "Copper(II) and molybdenum(VI) complexes of a tridentate ONN donor isothiosemicarbazone: Synthesis, characterization, X-ray, TGA and DFT", Polyhedron, vol. 115, pp. 297305, 5 September 2016.

[21] S. Rajković, M.D. Živković, B. Warżajtis, U. Rychlewska, et al, "Synthesis, spectroscopic and X-ray characterization of various pyrazinebridged platinum(II) complexes: $1 \mathrm{H}$ NMR comparative study of their catalytic abilities in the hydrolysis of methionine- and histidine-containing dipeptides", Polyhedron, vol. 117, pp. 367-376, 15 October 2016. 